STRUCTURAL BIOLOGY

ISSN 2059-7983

Keywords: new editors

\section{Three new Co-editors appointed to Acta Crystallographica Section D, Structural Biology}

\author{
Randy J. Read ${ }^{\mathrm{a} *}$ and Elspeth F. Garman ${ }^{\mathrm{b} *}$
}

a Department of Haematology, University of Cambridge, Cambridge Institute for Medical Research, The Keith Peters Building, Hills Road, Cambridge CB2 OXY, United Kingdom, and $\mathbf{b}$ Department of Biochemistry, University of Oxford, South Parks Road, Oxford OX1 3QU, United Kingdom. *Correspondence e-mail: rjr27@cam.ac.uk,

elspeth.garman@bioch.ox.ac.uk

We are delighted to welcome three new Co-editors to the editorial board of Acta Cryst. D. They enhance the already impressive range of expertise in our current board, and they help to reflect the diversity of our subject coverage, authors and readers.

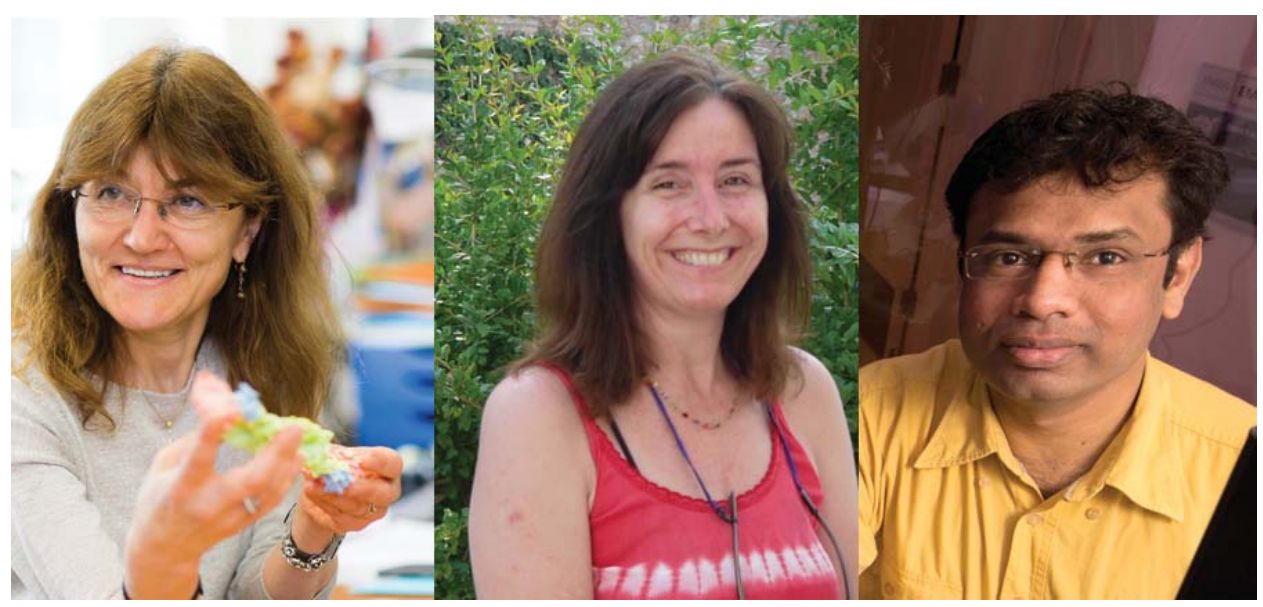

Kristina Djinović-Carugo is Head of the Department of Structural and Computational Biology in the Max Perutz labs in Vienna, Austria. With a major research interest in understanding the structural biology of complex systems such as the muscle Z-disk and the actin-based cytoskeleton, as well as of metalloproteins, she has developed expertise in integrative structural biology techniques that combine a variety of methods.

Ana González is the BioMAX beamline manager at the MAX IV synchrotron facility in Lund, Sweden. She brings thirty years of experience at a variety of synchrotrons around the world (Daresbury SRS, ESRF, EMBL Hamburg and SSRL before moving to Sweden) to the journal, with expertise in synchrotron science, radiation damage, optimal data collection for different phasing experiments, and the use of XFEL sources.

K. R. Vinothkumar is based at the National Centre for Biological Sciences of the Tata Institute of Fundamental Research in Bangalore, India. Prior to this, he developed expertise in cryo-EM techniques working with Werner Kühlbrandt at the MPI in Frankfurt, Germany and with Richard Henderson at the MRC-LMB in Cambridge, UK. His current research on membrane proteins and macromolecular complexes employs a combination of cryo-EM and X-ray diffraction, relevant to the growing number of cryoEM papers submitted to Acta Cryst. D and other IUCr journals.

We would also like to take this opportunity to thank all the people who are helping the journal to continue running smoothly during the COVID-19 crisis. The current board of co-editors have carried on dealing efficiently with submissions of new manuscripts. At the journal headquarters in Chester, Louise Jones and Simon Glynn have made the transition to working from home an impressively seamless operation.

Given the great emphasis on COVID-19-related research over this period (including a number of synchrotrons devoting data collection entirely to this area), we look forward to seeing new submissions addressing the structural biology of the virus and its interaction with the host, as well as our structural understanding of the disease pathology. 